\title{
Why are we still talking about open repair of descending aneurysms?
}

Allan Stewart, MD

From the Columbia University Medical Center, New York, New York.

Received for publication May 27, 2008; accepted for publication May 31, 2008

Address for reprints: Allen Stewart, MD Columbia University Medical Center, 177 Fort Washington Ave, MHB 7GN-435, New York, NY 10032 (E-mail: as2276@ columbia.edu)

J Thorac Cardiovasc Surg 2008;136:278-9

$0022-5223 / \$ 34.00$

Copyright (C) 2008 by The American Association for Thoracic Surgery

doi:10.1016/j.jtcvs.2008.05.021
$\mathrm{S}$ ince Alexander and Byron ${ }^{1}$ performed the first surgical intervention on a descending thoracic aneurysm in 1943, several advances have been made in the field. The conduit choice has moved from homografts ${ }^{2,3}$ to woven Dacron grafts. Methods to ameliorate heart strain and end-organ malperfusion syndrome progressed from surface hypothermia to arterial bypass shunts to partial left-sided heart bypass. Efforts to eliminate spinal ischemia have moved from "clamp and sew" to include lumbar drainage, administration of steroids, measurement of somatosensory evoked potentials, and evaluation of motor evoked potentials. These advancements, combined with an improvement in postoperative care, have resulted in a dramatic reduction in morbidity and mortality. In centers of excellence, a lower extremity paralysis rate less than $3.5 \%$, a stroke rate less than $3 \%$, and a mortality rate approximating $5 \%$ can be expected. ${ }^{4}$ Although open repair of descending thoracic aneurysms can be accomplished with a reproducibly low complication rate, it is an invasive procedure that requires a lengthy period of recovery.

Enthusiasm for a less-invasive solution began to gain traction with the successful endovascular repair of a thoracic aneurysm by Dake and colleagues. ${ }^{5}$ In 2005, the findings of the multicenter GORE TAG (WL Gore and Associates, Flagstaff, Ariz) study led to the US Food and Drug Administration approval for thoracic endovascular aortic repair (TEVAR). ${ }^{6}$ Initially, endovascular repair was limited to the elderly, patients with compromised functional status, and those with previous thoracic aortic interventions. Various medical subspecialties have since laid claim to the technology, including vascular surgeons, interventional radiologists, interventional cardiologists, and a small number of cardiac surgeons. The indications for stent grafting have yet to be completely defined, but examples include fusiform aneurysms, penetrating ulcers, complicated type B aortic dissection, coarctation of the aorta, and hybrid arch repair. Case reports and small series publications began to amass, with results claiming equivalence or superiority to open surgical repair. Comparisons with historical data demonstrated reductions in operative risk, hospital stay, and procedural cost. Despite an increasing literature, there is no prospective randomized study that compares endovascular repair with open surgery for isolated descending thoracic aneurysms. The available data confirm the feasibility of TEVAR, but confusion remains about the relative risk versus benefit of its widespread application. Ricco and colleagues ${ }^{7}$ published data from France, indicating that $17 \%$ of descending thoracic aortic aneurysms treated by stent grafting were placed in aneurysm smaller than $5.0 \mathrm{~cm}$, suggesting that the application of this technology is redefining the treatment paradigm or being administered inappropriately. As operator experience evolves and new generations of stents and delivery devices reach the market, excellent short-term results are reported and can be expected. However, now that follow-up has extended beyond 5 years, new complications are being reported, including endoleaks, stent migration, and stent fracture. With endografting seeming to be a bit less of a panacea, it is useful to review a large experience of open surgical repair to remind ourselves that open surgery may still be an option for some patients.

Minatoya and colleagues reported their 14 years experience with open repair of descending thoracic aneurysms. This article differs from those commonly found in the literature, because all of their patients have aortic anatomy that is amenable to endografting (ie, good proximal neck below the subclavian and above the celiac artery). Trials of prospective endografts seem to be delayed by the absence of such surgical 
controls, because these patients are commonly treated with endografting, despite the absence of long-term comparative data.

The article describes a surgical technique that differs from the commonly applied pure left-sided heart bypass, instead favoring femoral artery and venous cannulation coupled with an oxygenator. Flows were maintained at 1.5 to $2.0 \mathrm{~L} /$ $\mathrm{min}$, and temperature was allowed to drift. Motor evoked potentials were used to detect spinal ischemia, although spinal drainage was not used. Magnetic resonance imaging was implemented after 1998 to detect the artery of Adamkiewicz (AKA).

Overall results were reported $(\mathrm{n}=113)$, followed by a subgroup analysis comparing emergency and elective cases and subsequently assessing age as an independent variable on each subgroup. The early mortality rate was $5.3 \%$, with spinal cord dysfunction in $2.7 \%$ and a $7.1 \%$ rate of stroke. These data are in line with that demonstrated by major aortic centers throughout the United States. More remarkable, however, are the results demonstrated for the elective population $(n=97)$. Minatoya and colleagues report a $1 \%$ early mortality, $1 \%$ incidence of spinal dysfunction, and $4.1 \%$ incidence of stroke. They finally assert that no patient had a reintervention for the same lesion during the period of follow-up. These are impressive results and certainly should be applied as a metric when considering the modality of elective surgical correction of descending thoracic aortic aneurysms.

Equally important in this article are the results associated with open surgical correction of emergency cases $(n=16)$. The authors describe emergency cases as those who underwent repair in the setting of rupture. This cohort was not fleshed out further, but I suspect they had a contained rupture or some degree of extravasation of contrast on computed tomography. In this population, the results were poor with a mortality rate of $31.3 \%$, spinal injury in $12.5 \%$, and stroke rate of $25 \%$. The results were even worse in emergency cases in patients aged more than 75 years, in whom $22 \%$ had a spinal injury and $33.3 \%$ had a stroke.

The authors remarked at having located an AKA in 50 of 65 patients evaluated by magnetic resonance angiography. Although the artery was identified at operation in all of these patients, 2 patients had spinal cord dysfunction. These data suggest that the artery is present more frequently than given credit in the literature, but also remind us that more is involved in spinal perfusion than just the AKA.

Applying Minatoya and colleagues' data to daily practice, we have some useful data to stratify "good endograft" patients into a proper treatment modality. In the elective popu- lation, open surgery has excellent results for mortality (1\%) and spinal cord dysfunction (1\%). Equally important is the durability of repair with all patients having a freedom from reintervention during the entire time of follow-up. However, one must also refer to subgroup analysis when planning an open procedure for emergency repair. A mortality rate of $31 \%$ and a spinal cord injury rate of $22 \%$ are difficult to embrace. Far superior results have been reported in emergency cases treated with TEVAR (mortality: 7.9\%, spinal cord injury $1.7 \%$, stroke $3.7 \%){ }^{8}$ Perhaps the conclusion of the article should be modified to say that "the risks involved in replacement of the descending aorta under PCPB were comparable to those associated with thoracic endoprosthesis, in patients treated electively."

Endovascular therapy continues to emerge, and its offlabel use is limited only by the creativity of the operator. It is essential to carefully follow these patients to accurately assess the true impact of stent-graft therapy on the growth of aortic aneurysms. Comparable data are unlikely to dissuade practitioners from changing the course of therapy, especially true when the treating physician may not be capable of performing an open repair. Long-term follow-up, cost analysis (including surveillance cost and price of reintervention for endoleak), and quality of life metrics are essential to help guide the treating surgeon to recommend the best therapy for each patient. In the absence of such data, Minatoya and colleagues demonstrate that we still have a safe, durable, and reproducible operation to treat elective descending thoracic aneurysms.

\section{References}

1. Alexander J, Byron FX. Aortectomy for thoracic aneurysm. Univ Mich Hosp Bull. 1943;9:101.

2. Lam CR, Arom HH. Resection of descending thoracic aortic aneurysms without left heart bypass. Arch Surg. 1968;97:336.

3. DeBakey ME, Cooley DA. Successful resection of aneurysms of the thoracic aorta and replacement by graft. JAMA. 1953;152:673.

4. Coselli JS, LeMaire SA, Miller CC 3rd, et al. Mortality and paraplegia after thoracoabdominal aortic aneurysm repair: a risk factor analysis. Ann Thorac Surg. 2000;69:409.

5. Dake MD, Miller DC, Mitchell RS, et al. Transluminal placement of endovascular stent-grafts for the treatment of descending thoracic aortic aneurysms. N Engl J Med. 1994;331:1729.

6. Makaroun MS, Dillavou ED, Kee ST, et al. Endovascular treatment of thoracic aortic aneurysms: results of the phase II multicenter trial of the GORE TAG thoracic endoprosthesis. J Vasc Surg. 2005;41:1-9.

7. Ricco JB, Cau J, Marchand C, et al. Stent-graft repair for thoracic aortic disease: results of an independent nationwide study in France from 1999 to 2001. J Thorac Cardiovasc Surg. 2006;131:131-7.

8. Fatori R, Neinaber CA, Rousseau H, et al. Talent Thoracic Retrospective Registry. Results of endovascular repair of the thoracic aorta with the TALENT Thoracic Retrospective Registry. J Thorac Cardiovasc Surg. 2006;132:332-9. 